Y. C. Minh and E. F. van Dishoeck, eds.

\title{
ISO's View of the Molecular Content of Evolved Stars
}

\section{J. Cernicharo}

CSIC. IEM. Dpt. Molecular Physics, C/Serrano 121, 28006 Madrid, Spain

\begin{abstract}
The recent results of the ISO satellite in the field of molecular spectrocopy of AGB stars are reviewed. For the fist time, the two spectrometers onboard ISO have provided the opportunity to observe the pure rotational lines of several molecules in the far infrared and the rovibrational bands of the most abundant molecular species in the near and mid-infrared. These data allow to carry out a systematic study of the circumstellar envelopes of AGB stars and Planetary Nebulae. I analyze in this paper the role of resonant scatterring in the pumping of the ro-vibrational molecular levels in CSEs.
\end{abstract}

\section{Introduction}

While polar molecules can be detected at radio wavelengths, non-polar molecules can only be detected in the infrared through their active ro-vibrational transitions. On the other hand, observations of the vibrational bands of polar molecules complement the radio data providing essential constraints to the modelling of the observations, and to the determination of the physical and chemical conditions of the molecular gas in the circumstellar envelopes (CSE) of AGB stars. Some physical processes, like radiative pumping of molecular levels, which are very important in the energetic balance of the CSEs can be traced only at infrared wavelengths.

The ISO-SWS spectrometer (de Graauw et al. 1996) covers the 2.38-45 $\mu \mathrm{m}$ wavelength range, allowing a systematic search for the presence of nearly all likely abundant molecular species through their stretching and/or bending vibrational bands. The ISO-LWS spectrometer (Clegg et al. 1996) covers the 44-197 $\mu \mathrm{m}$ wavelength range and permits to observe the pure rotational lines of species like $\mathrm{CO}, \mathrm{H}_{2} \mathrm{O}, \mathrm{HCN}, \ldots$ The spectral resolution provided by the SWS full resolution grating observations, $R=1500-2000$, permits to resolve the rotational structure of the molecular bands arising from molecules with rotational constants larger than $0.2 \mathrm{~cm}^{-1}$ - i.e. from practically all diatomic and triatomic species and from some four-atomic molecular species. The SWS and LWS complement the already available ro-vibrational data obtained from ground based telescopes and allow the study of vibrational bands and pure rotational lines for which the observation from ground-based telescopes is difficult, or even impossible, due to the low atmospheric transmission at these wavelengths. 


\section{The Warm Layers of the Circumstellar Envelopes of AGB Stars}

In comparison with the millimeter/submillimeter domains where up to now most of the studies have been made, the near-, mid- and far-IR range provide a unique opportunity to probe the inner hottest regions of the CSE. The observation of molecular transitions involving high quantum numbers at far-IR wavelengths and the observation of hot- and combination-bands in the near and mid-infrared allow to trace the molecular material in the innermost zones of the CSE. Tsuji et al. (1997) have analyzed the ISO-SWS spectra in the direction of M giants and Miras. They found that the stretching band of water vapour at $2.7 \mu \mathrm{m}$ and the $\mathrm{CO}_{2}$ stretching band at $4.2 \mu \mathrm{m}$ appear in absorption in these objects. The analysis of these molecular bands indicates excitation temperatures around $750-1250 \mathrm{~K}$. Tsuji et al. (1997) suggest that these features are produced in a rather warm molecule-forming region above the photosphere that could be related to the quasi-static molecular zone previously observed from high resolution observations of $\mathrm{CO}, \mathrm{C}_{2} \mathrm{H}_{2}$ and other molecular species (Keady \& Hinkle 1988; Keady \& Ridway 1993). This warm molecular zone is very close to the star and has also been observed in the near and mid-IR ISO's spectrum of IRC+10216 (Cernicharo et al. 1999a; see below). Yamamura et al. (1999a) have observed $\mathrm{SO}_{2}$ in some O-rich objects. They have modelled the observations with very simple assumptions that allow to reproduce the shape of the band as a function of the stellar phase. They conclude that $\mathrm{SO}_{2}$ is very abundant in the innermost regions of O-rich CSEs and that the different ISO data indicate the presence of several warm layers of high density which could be related to the dynamical processes associated with stellar pulsation. However, their models are too simple because they neglect some important physical processes, like radiative pumping effects, on the population of the ro-vibrational levels of these molecular species (González-Alfonso \& Cernicharo 1999a,b). We will discuss these effects in the sections devoted to $\mathrm{HCN}$ in $\mathrm{IRC}+10216$ and to $\mathrm{H}_{2} \mathrm{O}$ in O-rich stars.

\section{Carbon-rich AGB Stars: The Case of IRC+10216}

IRC+10216 is the brightest C-rich evolved object in the sky and is the prototype of C-rich AGB objects. It has an extended CSE where around 50 molecular species have been detected, and it is probably one of the best studied stellar objects at infrared and radio wavelengths (Cernicharo, Guélin, \& Kahane 2000). IRC +10216 has a particularly rich carbon chemistry, in such a way that most of the species detected in this source are carbon chain radicals $\left(\mathrm{C}_{5} \mathrm{H}\right.$ : Cernicharo et al. 1986a,b, 1987a; $\mathrm{C}_{6} \mathrm{H}$ : Suzuki et al. 1986, Guélin et al. 1987, Cernicharo et al. 1987b; $\mathrm{C}_{8} \mathrm{H}$ : Cernicharo \& Guélin 1996; $\mathrm{C}_{7} \mathrm{H}$ : Guélin et al. 1997; $\mathrm{H}_{2} \mathrm{C}_{3}$ and $\mathrm{H}_{2} \mathrm{C}_{4}$ : Cernicharo et al 1991a,b; $\mathrm{C}_{5} \mathrm{~N}$ : Guélin, Neininger, \& Cernicharo 1998) which are formed in the external layers of the CSE (Guélin, Lucas, \& Cernicharo 1993). Other species of interest detected in IRC+10216 include silicon carbide (SiC; Cernicharo et al. 1989a), the metal-bearing molecules, such as the metal halides $\mathrm{NaCl}, \mathrm{AlCl}, \mathrm{AlF}$, and $\mathrm{KCl}$, detected for the first time in this CSE by Cernicharo \& Guélin (1987), NaCN (Turner, Steimle, \& Meerts 1994). Some metal-bearing species were also detected in the external shell of IRC+10216: MgNC (Ishii et al. 1993; Guélin et al. 1986; Guélin, Lucas, \& Cernicharo 1993) and MgCN (Ziurys 


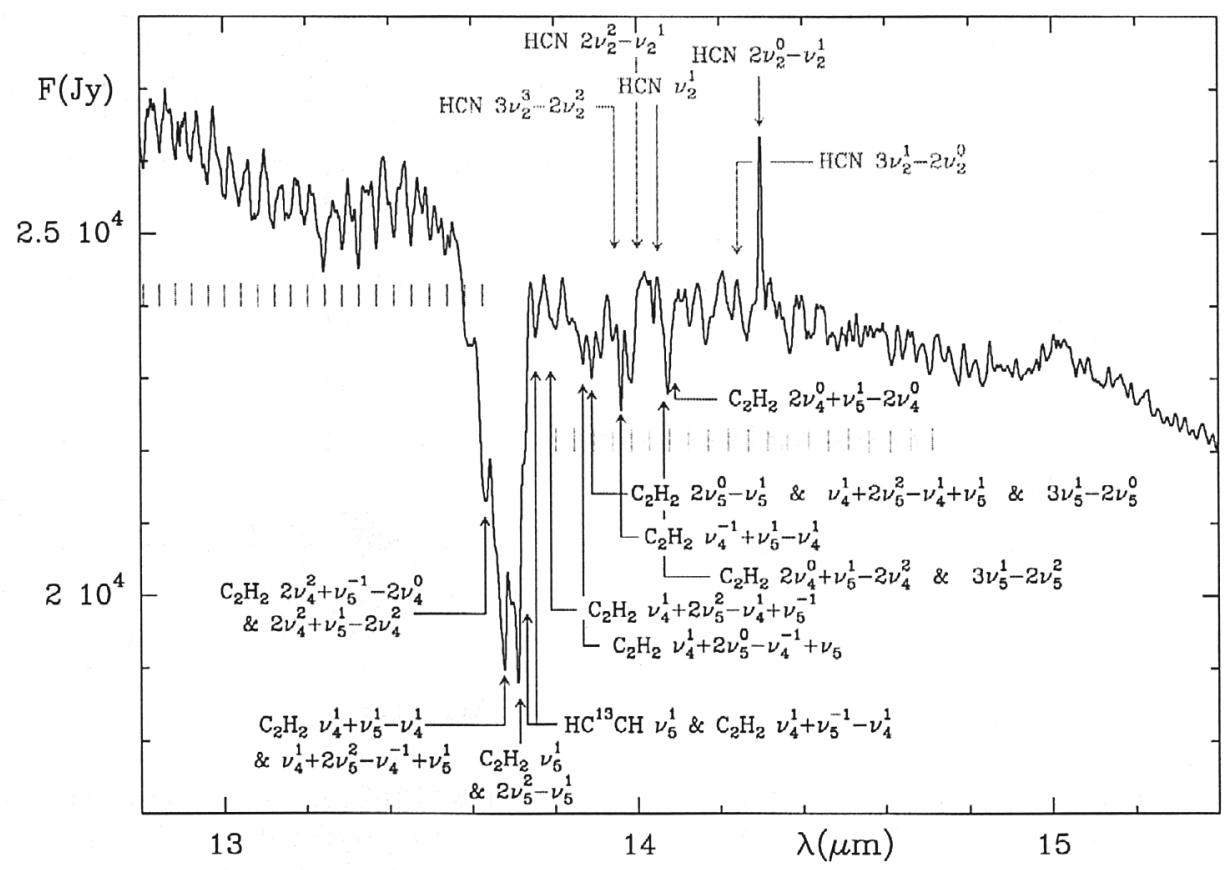

Figure 1. Observed ISO-SWS spectrum of IRC+10216 around 15 $\mu \mathrm{m}$. Spectral resolution is $\simeq 200 \mathrm{~km} \mathrm{~s}^{-1}$. The positions of the bands are indicated. Vertical lines indicated the position of the individual $\mathrm{R}$ and $\mathrm{P}$ lines of the fundamental bending mode of $\mathrm{C}_{2} \mathrm{H}_{2}$. Note that the $\mathrm{HCN}$ bands are in emission as indicated by the shape of the $\mathrm{P}$-band of $\mathrm{C}_{2} \mathrm{H}_{2}$ relative to the $\mathrm{R}$ one and by the strong Q-band of the $2 \nu_{2} l=0-$ $\nu_{2} l=1$ transition.

et al. 1995). The emission from the innermost regions of the CSE of IRC+10216 seems to indicate a chemistry in thermodynamical equilibrium (Tsuji 1973). Most of the stable diatomic, triatomic and also many polyatomic species are formed efficiently in these layers $\left(\mathrm{CO}, \mathrm{HCN}, \mathrm{C}_{2} \mathrm{H}_{2}, \mathrm{SiO}, \mathrm{CS}, \mathrm{SiS}, \ldots\right)$.

\subsection{The near and mid-infrared spectrum of IRC+10216}

Cernicharo et al. (1999a) have observed IRC+10216 with the ISO-SWS spectrometer. They have found a broad absorption feature at $3 \mu \mathrm{m}$ which is due to the stretching modes of $\mathrm{C}_{2} \mathrm{H}_{2}$ and $\mathrm{HCN}$. In addition, the Q-branches of the $\nu_{3}+\nu_{5}, \nu_{3}+\nu_{4}$ and $\nu_{2}+2 \nu_{4}+\nu_{5}$ modes of $\mathrm{C}_{2} \mathrm{H}_{2}$ are detected around $2.5 \mu \mathrm{m}$. Many combination bands of the same molecule, like $\nu_{2}+\nu_{5}$ among others, are detected around $3.8 \mu \mathrm{m}$. The Q-branch of the $\nu_{2}+\nu_{3}$ mode of HCN and those of the $\nu_{1}+\nu_{2}$ and $\nu_{1}+2 \nu_{2}-\nu_{2}$ vibrational transitions of HCN are detected in absorption around $2.5 \mu \mathrm{m}$ and $3.6 \mu \mathrm{m}$ respectively. The $\nu_{3}-\nu_{2}$ mode of HCN and other combination bands are also detected (see their Figure 2). By assuming an LTE distribution of the $\mathrm{C}_{2} \mathrm{H}_{2}$ and $\mathrm{HCN}$ level populations, they modelled the absorption produced by 21 bands of $\mathrm{C}_{2} \mathrm{H}_{2}$ and 2 bands of $\mathrm{H}^{13} \mathrm{CCH}$, together with three bands of HCN and the $\nu_{3}$ band of $\mathrm{H}^{13} \mathrm{CN}$. Cernicharo et al. (1999a) conclude 


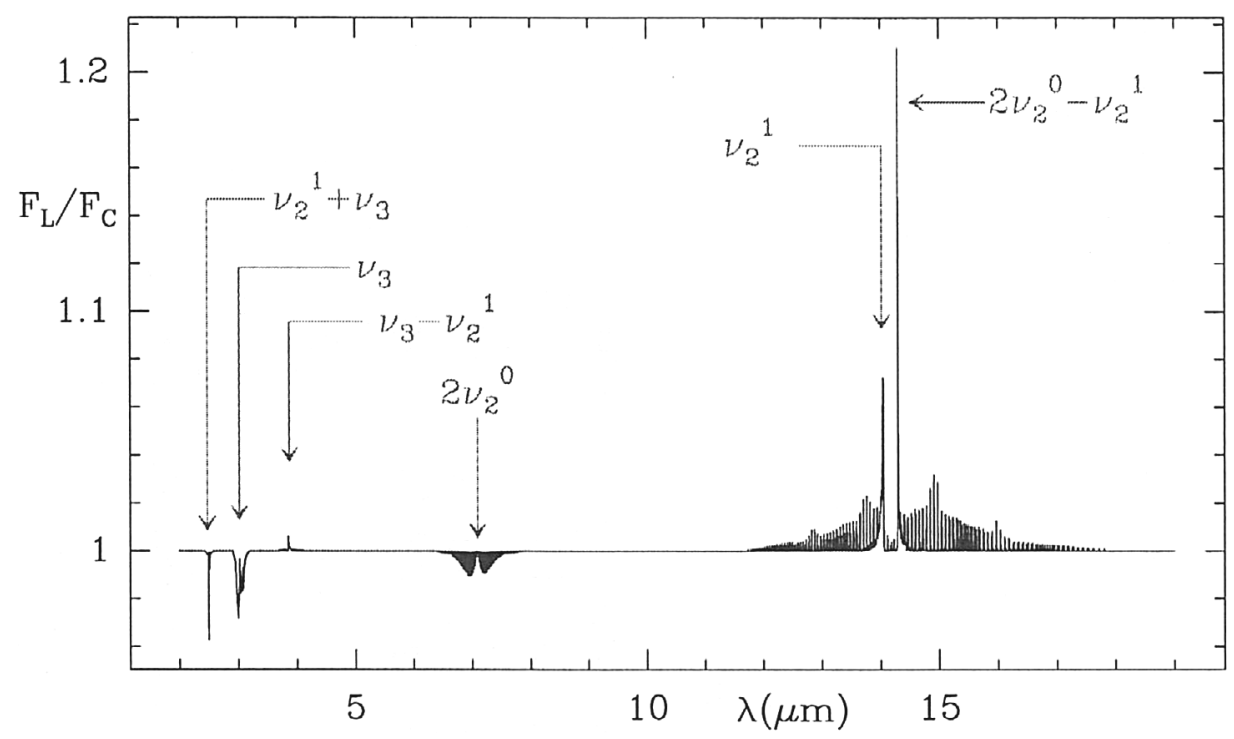

Figure 2. Modelled HCN spectrum in IRC+10216. The model is described in the text. Note the large intensity predicted for the $\mathrm{Q}^{-}$ band of the $2 \nu_{2} l=0-\nu_{2} l=1$ transition in good agreement with the observations. In addition, the $(0,0,1)-\left(0,1^{1}, 0\right)$ band is also predicted in emission (from Cernicharo et al. 1999a and González-Alfonso \& Cernicharo 1999a).

that a model with a single layer cannot explain the observed band profile (see Keady \& Hinkle 1988). They adopted the detailed CSE structure derived by Keady \& Hinkle and $\mathrm{X}\left(\mathrm{C}_{2} \mathrm{H}_{2}\right)=510^{-5}$ and $\mathrm{X}(\mathrm{HCN})=310^{-5}$ (from Cernicharo et al. 1996, Wiedemann et al. 1991; see below). In order to fit the wings of the $3 \mu \mathrm{m}$ feature they needed to include an additional contribution from warm gas in the inner region $\left(T_{K} \approx 1700 \mathrm{~K} ; 1<\mathrm{r}<3 \mathrm{R}_{*}\right)$. This layer could be similar to the warm molecular envelope found by Tsuji et al. (1997) in other CSEs (see also above) and its origin is probably related to the pulsation of the central star (Woitke et al. 1999).

Figure 1 shows the $14 \mu \mathrm{m}$ spectrum of IRC+10216 (Cernicharo et al. 1999a). The absorption features are due to the $\nu_{5}$ bending mode of acetylene and its associated hot and combination bands. This figure shows that the HCN bands appear in emission with the Q-branch of the $2 \nu_{2}^{0}-\nu_{2}^{1}$ transition being particularly strong. The HCN emission has to be modelled in a more complicated way than $\mathrm{C}_{2} \mathrm{H}_{2}$. The $\nu_{3}$ band of $\mathrm{HCN}$ at $3 \mu \mathrm{m}$ is seen in absorption (Cernicharo et al. 1999a) whereas the bending states are detected in emission against the continuum. P-Cygni profiles are in fact expected for $\mathrm{HCN}$ and $\mathrm{C}_{2} \mathrm{H}_{2}$ (Wiedemann et al. 1991). The larger beam size of the SWS compared to the high angular resolution data of Wiedemann et al. could favour the observation of re-emission from $\mathrm{HCN}$ through resonant scattering in the molecular envelope.

The different behaviour of the bending bands of $\mathrm{C}_{2} \mathrm{H}_{2}$ and $\mathrm{HCN}$ must be related to the larger abundance of $\mathrm{C}_{2} \mathrm{H}_{2}$ through the whole envelope (which in- 
creases the importance of stellar blocking, and absorption by the dust due to the larger number of absorption and re-emision processes; see González-Alfonso \& Cernicharo 1999a,b) and/or to the excitation mechanism that gives rise to the HCN band emission. Cernicharo et al. conclude that an important difference between both molecules would be established if rovibrational excitation is mainly radiative, instead of collisional: while the $2 \nu_{2}^{0}$ state of $\mathrm{HCN}$ is radiatively connected to the ground state, the equivalent transition in $\mathrm{C}_{2} \mathrm{H}_{2}$ is forbidden, i.e., no photons can be absorbed from the ground to the $2 \nu_{5}^{0}$ state due to the fact that both levels have the same symmetry. In HCN, photons absorbed from the ground to the $2 \nu_{2}^{0}$ level will decay preferently to the $\nu_{2}^{1}$ level, because the Einstein coefficient for spontaneous emission of this transition is $\sim 4 \mathrm{~s}^{-1}$, i.e., twice the value corresponding to the decay to the ground state. The equivalent process in $\mathrm{C}_{2} \mathrm{H}_{2}$ is radiative excitation from the ground to the $\nu_{4}+\nu_{5}$ state followed by radiative decay to the $\nu_{4}$ level. However, this transition is very close in frequency to the Q-branch of the $\nu_{5}$ transition, and any possible reemission could not compensate the strong absorption from the ground to the $\nu_{5}$ bending mode.

By using the non-local, non-LTE code developed by González-Alfonso \& Cernicharo (1997), Cernicharo et al. (1999a) have modelled the HCN emission/absorption in IRC+10216. The results (see Figure 2) predict that the $\nu_{3}, \nu_{3}+\nu_{2}$ and the $2 \nu_{2}$ bands of HCN will be in absorption while the $\nu_{2}$ and its overtone bands will be in emission. The model also predicts that the $\nu_{3}-\nu_{2}$ band will be in emission as observed by Cernicharo et al. The best fit to the data was obtained with an HCN abundance of $\sim(1-1.5) 10^{-5}$ which is only a factor of two smaller than that derived from the ISO-LWS data (see below). The pumping mechanisms are different for the different regions of the envelope. In the innermost regions, before the dust formation zone, absorption of photons at $3 \mu \mathrm{m}$ in the $\nu_{3}$ mode will be very efficient. Radiative decay to the $2 \nu_{2} l=0$ and $\nu_{2} l=1$ states will be an important mechanism to populate both levels. Also in this region the $\nu_{3}-\nu_{2}$ band will appear in emission. Outside the dust formation region the flux at $3 \mu \mathrm{m}$ decreases but it increases considerably at $7.1 \mu \mathrm{m}$. The pumping from the ground state to the $2 \nu_{2} l=0$ vibrational level, followed by radiative decay to the $\nu_{2} l=1$ level, will be the main mechanism in producing the observed emission at $14.3 \mu \mathrm{m}$. Consequently, the intensity of the HCN bands, as observed by ISO, could be very different in stars with low, moderate or high mass loss rates (see, e.g. Aoki et al. 1999). These calculations show that resonant scattering through the envelope is very efficient for HCN and that it could also have an effect on the heating and cooling of the molecular gas (Cernicharo 1998).

\subsection{The far-infrared spectrum of IRC $+\mathbf{1 0 2 1 6}$}

Cernicharo et al. (1996) have observed the 43-197 $\mu \mathrm{m}$ spectrum of IRC+10216 as observed with the ISO-LWS. Figure 3 shows the continuum-subtracted LWS spectrum of IRC+10216 together with the results of a LVG model for CO and HCN (Cernicharo et al. 1996). The spectrum is dominated by the emission of carbon monoxide and $\mathrm{HCN}$ in all their vibrational states. Some lines of ${ }^{13} \mathrm{CO}$ and $\mathrm{H}^{13} \mathrm{CN}$ are also detected. The $\mathrm{CO}$ lines are strong throughout the wavelength range of the LWS and only show a decrease in the intensity for the highest $\mathrm{J}$ 


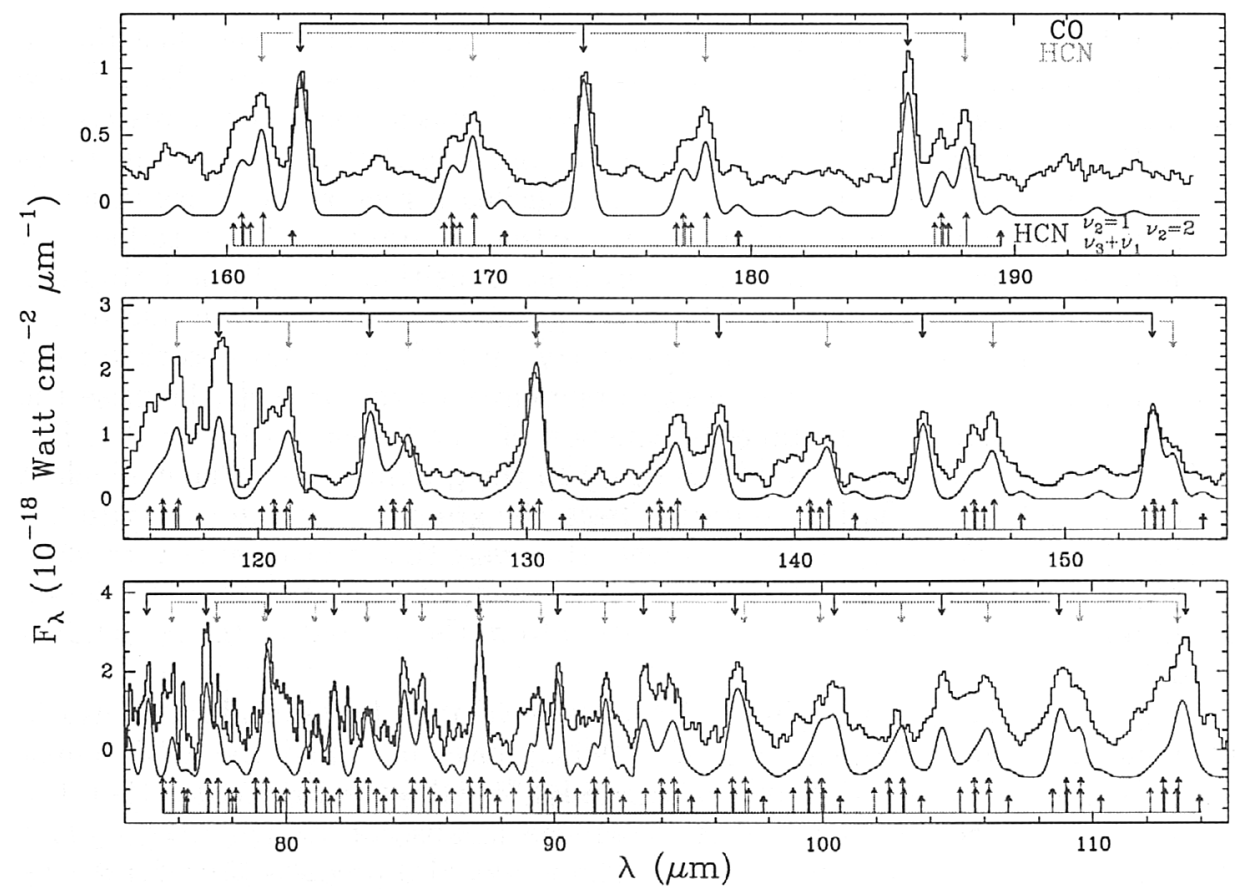

Figure 3. Continuum subtracted LWS grating spectrum of IRC+10216 from 75 to $197 \mu \mathrm{m}$ (thick line). An offset has been introduced to the continuum to show simultaneously the computed emission of the $\mathrm{CO} v=0,1,{ }^{13} \mathrm{CO} v=0, \mathrm{HCN}$ and $\mathrm{H}^{13} \mathrm{CN} v=0$, and $\mathrm{HCN} \nu_{2}=1,2$ $\nu_{1,3}=1$ (thin line). The rotational transitions of $\mathrm{CO}, \mathrm{HCN}$, and $\mathrm{HCN}$ $\nu_{2}=1,2$ and $\nu_{1,3}=1$ are indicated at the top and bottom of each panel. The weak lines in the model correspond to $\mathrm{H}^{13} \mathrm{CN},{ }^{13} \mathrm{CO}$ and to the stretching modes of HCN (from: Cernicharo et al. 1996).

rotational transitions. Besides the rotational lines of $\mathrm{CO}$, a forest of HCN lines from different vibrational states were detected from $J=18 \rightarrow 17$ to $48 \rightarrow 47$. HCN has a bending mode, $\nu_{2}$, at $\simeq 713 \mathrm{~cm}^{-1}$, and two stretching modes $\nu_{1}$ and $\nu_{3}$, at 2096 and $3311 \mathrm{~cm}^{-1}$ respectively. Cernicharo et al. (1996) concluded that after removing the $\mathrm{CO}$ lines, all the remaining strong features in the far-IR spectrum of IRC+10216 are due to HCN. Some of the weak features remaining in the far-IR spectrum of IRC+10216 could be assigned to rotational transitions of CS and $\mathrm{SiO}$. However, the limited spectral resolution of the data in Figure 3 avoids any certain identification for these features. Cernicharo et al. (1996) searched for $\mathrm{NaH}, \mathrm{MgH}, \mathrm{CaH}, \mathrm{NH}, \mathrm{CH}, \mathrm{FeH}, \mathrm{NiH}, \mathrm{SH}, \mathrm{SiH}, \mathrm{CH}_{2}$, and other light molecular species without success. However, they reported a tentative detection of the $\mathrm{Q}(2,4,6,8)$ lines of the bending mode of triatomic carbon, $\mathrm{C}_{3}$, around $157.2 \mu \mathrm{m}$ (Cernicharo et al., in preparation). Some weak and broad features present in Figure 3 could also correspond to the $\nu_{2}=1,2$ levels of $\mathrm{H}^{13} \mathrm{CN}$.

The most spectacular result from the ISO-LWS spectrum of IRC+10216 is the forest of lines arising from the different vibrational levels of HCN. This result could have been expected on the basis of the strong maser emission found by 

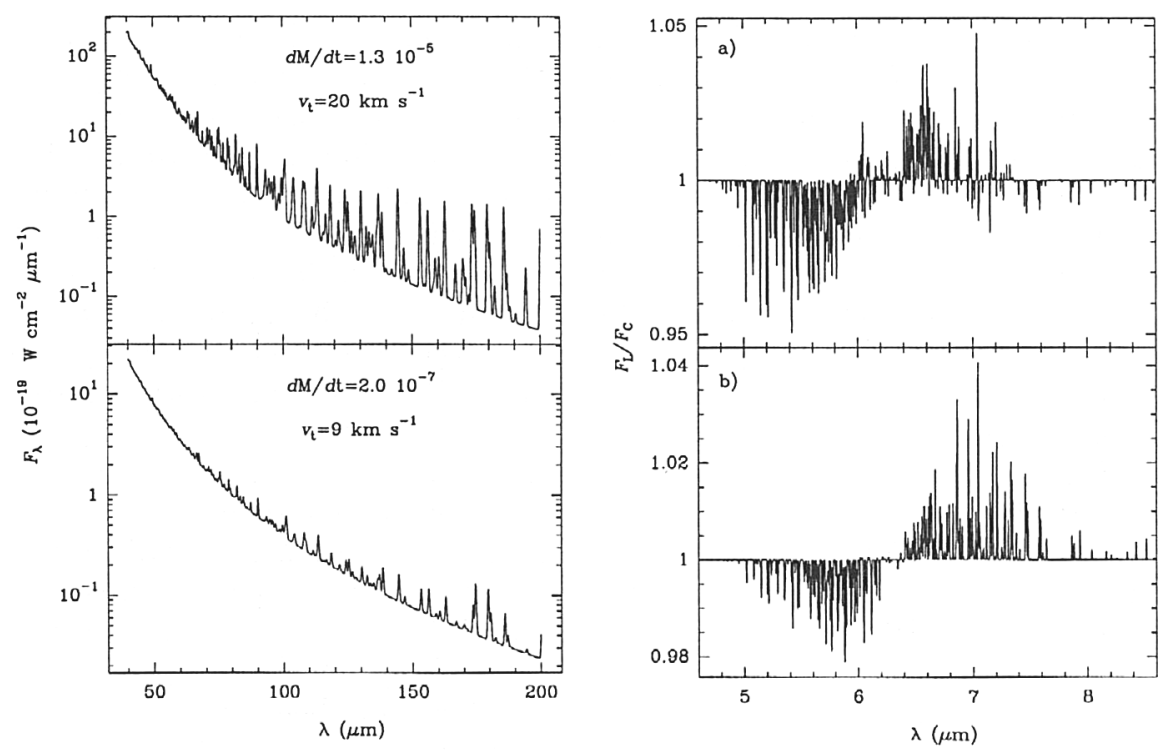

Figure 4. (left panels) Expected LWS spectrum in O-rich stars for high (upper panel) and low mass loss rates (lower panel). The models include the the pure rotational lines of $\mathrm{H}_{2} \mathrm{O}$ and $\mathrm{CO}$. (right panels) Expected SWS spectrum around $6 \mu \mathrm{m}$ for the two models shown in the left panels (González-Alfonso \& Cernicharo 1999b). The predicted behaviour of the bending mode of water vapour has been observed in the direction of high mass star-forming regions (González-Alfonso et al. 1998).

Lucas \& Cernicharo (1989) in the $\nu_{2}=1$ state and the strong thermal emission in the $J=2 \rightarrow 1$ and $J=3 \rightarrow 2$ lines from the other vibrational levels of HCN. The best agreement between the observations and the model results (which are shown in Figure 3 ) is obtained for $\mathrm{HCN} / \mathrm{CO}=0.1$ which corresponds to an $\mathrm{HCN}$ abundance of $310^{-5}$, a value that agrees with that derived from millimeter observations (Cernicharo et al. 1987a). Taking into account the uncertainties associated with the data calibration and with the baseline used to remove the continuum, the agreement between the observations and our model results is reasonably good. In the far-IR, $\lambda>70 \mu \mathrm{m}$, the power emitted in the HCN lines is $0.44 \mathrm{~L}_{\odot}$ while that of $\mathrm{CO}$ is $0.28 \mathrm{~L}_{\odot}$ (even when the millimeter and sub-millimeter lines of $\mathrm{CO}$ and $\mathrm{HCN}$ are included). Hence $\mathrm{HCN}$ is the main coolant in the inner regions of this C-rich CSE. HCN plays in C-rich AGB stars a similar role to that of water in O-rich CSEs. Ryde et al. (1999a) have also observed the CO far-IR lines with ISO in the C-rich object IRAS15194-5115.

\section{Oxygen-rich AGB Stars}

About 30 pure rotational lines of water vapour have been detected in the oxygenrich AGB star W Hya by Barlow et al. (1996). A similar study of R Cas has been carried out by Truong-Bach et al. (1999). Unlike the spectrum of IRC+10216, 
the far-infrared spectrum of O-rich stars is dominated by the emission of the rotational lines of $\mathrm{H}_{2} \mathrm{O}$. Taking into account the large variation of the temperature and density profiles across the envelopes, these lines, which cover a very large range of energies and Einstein coefficients, could provide the best tool to derive the physical conditions of the gas in these objects. The water vapour abundance derived from the modelling of the data in W Hya is $10^{-4}$. Neufeld et al. (1996) have observed 4 high excitation transitions of $\mathrm{H}_{2} \mathrm{O}$ with the SWS-FP spectrometer in the same object but reached different values for the mass loss rate of the star due to the different temperature profile adopted in their modelling, which shows the strong dependence of the intensity of the $\mathrm{H}_{2} \mathrm{O}$ pure rotational lines with the physical conditions in the CSE of O-rich objects.

The ro-vibrational transitions of the bending mode of $\mathrm{H}_{2} \mathrm{O}$, together with the stretching mode of $\mathrm{OH}$ between $3-3.5 \mu \mathrm{m}$ and the stretching mode of $\mathrm{CO}_{2}$ at $4.2 \mu \mathrm{m}$, were observed for the first time in the evolved star NML Cyg by Justtanont et al. (1996) using the SWS spectrometer. Far-IR OH lines have been observed in IRC+10420 by Sylvester et al. (1997). They analyse the role of these lines in the pumping of the $\mathrm{OH}$ maser transitions.

ISO-SWS data of large sample of O-rich stars have been analyzed by Matsuura et al. (1999). Yamamura et al. (1999b) have observed the stretching mode of water vapour in Mira and in Z Cas. In the former object they found the band in emission while in the latter it is observed in absorption. Using an LTE approximation they conclude that the different behaviour of the vibrational band is due to the different size of the $\mathrm{H}_{2} \mathrm{O}$ warm layer around the central star. The densities needed to fill the LTE assumption, like in the case of $\mathrm{CO}_{2}$ (Justtanont et al. 1998; Ryde et al. 1999b) and of $\mathrm{SO}_{2}$ (Yamamura et al. 1999b), are very high but roughly consistent with dynamical model atmospheres (Woitke et al. 1999). These LTE plane-parallel models are in fact very simple and, due to the lack of spectral resolution, depend on only three parameters: the excitation temperature, the size of the molecular layer and the molecular abundance. However, they neglect all pumping mechanisms other than collisions (which they assume large enough to quasi-thermalize the vibrational levels). Due to the selection rules associated with vibrational transitions prevailing for the bending and overtone modes of triatomic molecules, these models cannot account for some of the observed properties of the $\mathrm{H}_{2} \mathrm{O}, \mathrm{CO}_{2}$ and $\mathrm{HCN}$ (see above) and in particular for the important role of radiative pumping through the absorption of photons from the central object and its surrounding dust. González-Alfonso \& Cernicharo (1999a,b) have modelled the $\mathrm{H}_{2} \mathrm{O}$ emission in O-rich stars with the use of non-LTE and non-Local codes that allow to treat simultaneously dust and molecules. Figure 4a shows the computed far-IR spectrum (pure rotational lines) for two different mass loss rates and terminal velocities. In both cases the distance to the star is $500 \mathrm{pc}$ and the water vapour abundance is assumed to be $2.510^{-4}$. The radius of the star and of the envelope are $3.510^{13}$ and $10^{17}$ $\mathrm{cm}$ respectively. The stellar temperature is $2500 \mathrm{~K}$. The spectrum shown in the bottom panel could apply to W Hya (see Barlow et al. 1996) when the fluxes are corrected for the distance of this object $(100 \mathrm{pc})$. The top panel of Figure $4 \mathrm{a}$ applies for a star with very large mass loss rate like VX Sgr or VY CMa (after a distance correction). The $\mathrm{CO}$ lines are much weaker than those of $\mathrm{H}_{2} \mathrm{O}$ for the mass loss rate of $\mathrm{W}$ Hya but become comparable to the $\mathrm{H}_{2} \mathrm{O}$ lines for $d M / d t$ as 


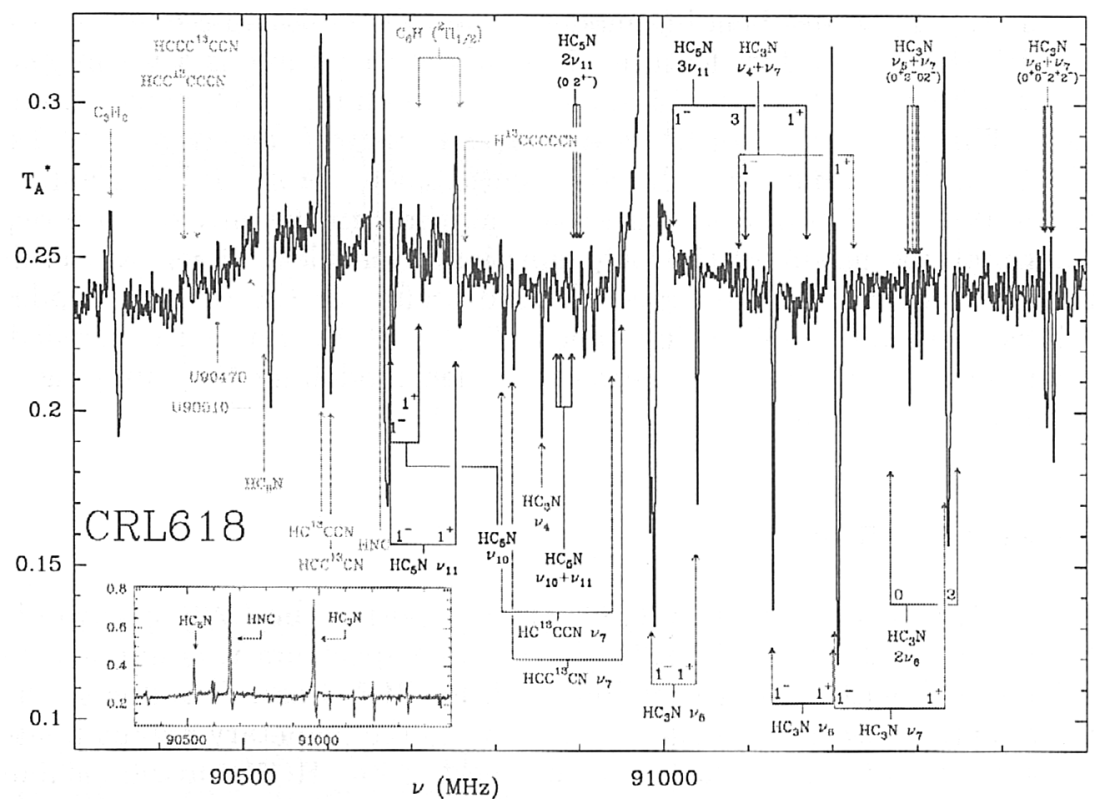

Figure 5. The spectrum of CRL618 around $91000 \mathrm{MHz}$ as observed with the 30-m IRAM radio telescope. Most of the absorption lines are due to ro-vibrational lines of $\mathrm{HC}_{3} \mathrm{~N}$ and $\mathrm{HC}_{5} \mathrm{~N}$.

high as $10^{-5} \mathrm{M}_{\odot} \mathrm{yr}^{-1}$. The cooling of the gas is dominated in these objects by the emission of pure rotational lines of water vapour.

Figure $4 \mathrm{~b}$ shows the results for the bending mode of $\mathrm{H}_{2} \mathrm{O}$ at $6 \mu \mathrm{m}$. Some rovibrational lines will be seen in absorption while many others will be in emission. Resonant scattering through the envelope is responsible for this behaviour. For stars with very high mass-loss rates the band will be seen in absorption. However, for medium and low mass loss rates the band will have a profile similar to the plots in Figure 4b (see González-Alfonso \& Cernicharo 1999a,b for details). Resonant scattering will have a very important role in the heating of the gas in the region where collisions between the vibrational levels are negligible but where collisions inside the ground state can repopulate its rotational levels. A photon absorbed in the ground level will pump the molecule to a given level in the bending vibrational level. Reemission to one of the allowed rotational levels in the ground state, followed by collisional de-excitation in the ground state, will put energy in the gas phase. The region where this mechanism applies corresponds to that of densities larger than a few $10^{5} \mathrm{~cm}^{-3}$, but low enough to have negligible vibrational excitation through collisions. In stars with low mass loss rate this region is located near the photosphere while in stars with very high mass loss rate it starts at $10-20$ stellar radii.

In the near infrared Justtanont et al. (1996) have reported the detection of $\mathrm{CO}_{2}$ in gas phase at $4.3 \mu \mathrm{m}$ in NML Cyg. In the same object they have also identified the bending and stretching modes of $\mathrm{H}_{2} \mathrm{O}$. Several pure rotational lines were also observed in emission. Recently, Justannont et al. (1998) have discovered strong $\mathrm{CO}_{2}$ emission around $15 \mu \mathrm{m}$ corresponding to vibrational transitions 
between stretching and bending modes. The $\mathrm{CO}_{2}$ emission seems to be correlated with the $13 \mu \mathrm{m}$ dust feature. They argue that there is a warm gas layer $(650-1250 \mathrm{~K})$ where both the $13 \mu \mathrm{m}$ feature dust and the $\mathrm{CO}_{2}$ emission lines are formed. The existence of such a warm layer has also been claimed by Tsuji et al. (1997) in their study with the SWS spectrometer of several M giants. However, detailed analysis of resonant scattering through the envelope are required to determine the nature of the $\mathrm{CO}_{2}$ emission. González-Alfonso \& Cernicharo (1999a) have computed the expected band-profile for the bending modes and associated hot- and combination-bands of $\mathrm{CO}_{2}$ at $15 \mu \mathrm{m}$ and concluded that resonant scattering cannot be neglected when modelling the pumping of the ro-vibrational bands of $\mathrm{CO}_{2}$.

\section{Post-AGB Stars}

Cox et al. (1996) and Liu et al. (1996) have reported the LWS spectra of CRL 2688 and NGC7027. The CRL2688 far-infrared spectrum is dominated by the emission of $\mathrm{CO}$ with a weak contribution from HCN lines. It is due to the fact that the central star has started its evolution to the planetary nebula phase and that the innermost part of the envelope, where strong $\mathrm{HCN}$ emission is found in IRC+10216, has been removed. In NGC7027 the emission is completely dominated by $\mathrm{CO}$ and atomic fine structure lines. The high velocity wind associated with the evolution to the planetary phase and the UV photons from the central bright star perturb the circumstellar envelope in these objects producing shocks and photodissociation regions (PDRs) which modify the physical and chemical conditions of the gas (Cernicharo et al. 1989a; Neri et al. 1992; Herpin \& Cernicharo 1999).

CRL618 is a C-rich star evolving very fast towards the planetary nebula stage. The molecular content of the circumstellar envelope of this object, as observed at millimeter wavelengths, is very similar to that of IRC+10216, i.e., long carbon radicals like $\mathrm{C}_{4} \mathrm{H}$ and $\mathrm{C}_{6} \mathrm{H}$, cyanopolyynes such as $\mathrm{HC}_{3} \mathrm{~N}, \mathrm{HC}_{5} \mathrm{~N}$, $\mathrm{HC}_{7} \mathrm{~N}$, and $\mathrm{HC}_{9} \mathrm{~N}$, etc. However, its millimeter spectrum presents a remarkable difference with respect to IRC+10216: pure rotational lines of highly excited vibrational states of $\mathrm{HC}_{3} \mathrm{~N}$ and $\mathrm{HC}_{5} \mathrm{~N}$ produce a forest of absorption lines that are not present in IRC+10216 (Cernicharo et al. 1999b and Figure 5). Only the low energy $\nu_{7}$ mode of $\mathrm{HC}_{3} \mathrm{~N}$ and perhaps the lowest energy $\nu_{11}$ mode of $\mathrm{HC}_{5} \mathrm{~N}$ are detected in IRC+10216. CRL618 also presents an extremely high velocity wind, covering $400 \mathrm{~km} \mathrm{~s}^{-1}$ (Cernicharo et al. 1989b), that perturbs the CSE from its AGB phase producing shocked regions, where molecules can be destroyed and reformed under very different physical conditions than those prevailing in the AGB phase (see Cernicharo et al. 1989a,b; Neri et al. 1992). As a consequence of this activity, CRL618 is the only C-rich object where $\mathrm{H}_{2} \mathrm{CO}$ has been detected (see Cernicharo et al. 1989a,b).

Although the difference in the spectrum between both objects can be explained as due to different physical conditions, the millimeter data indicate the presence of molecules that are not detected in IRC+10216: $\mathrm{CH}_{3} \mathrm{C}_{2} \mathrm{H}$ and $\mathrm{CH}_{3} \mathrm{C}_{4} \mathrm{H}$. Moreover, the column densities for the long cyanopolyynes are higher than in IRC+10216. The infrared spectrum of both sources could reveal the richness of their molecular content and of their physical and chemical conditions. 


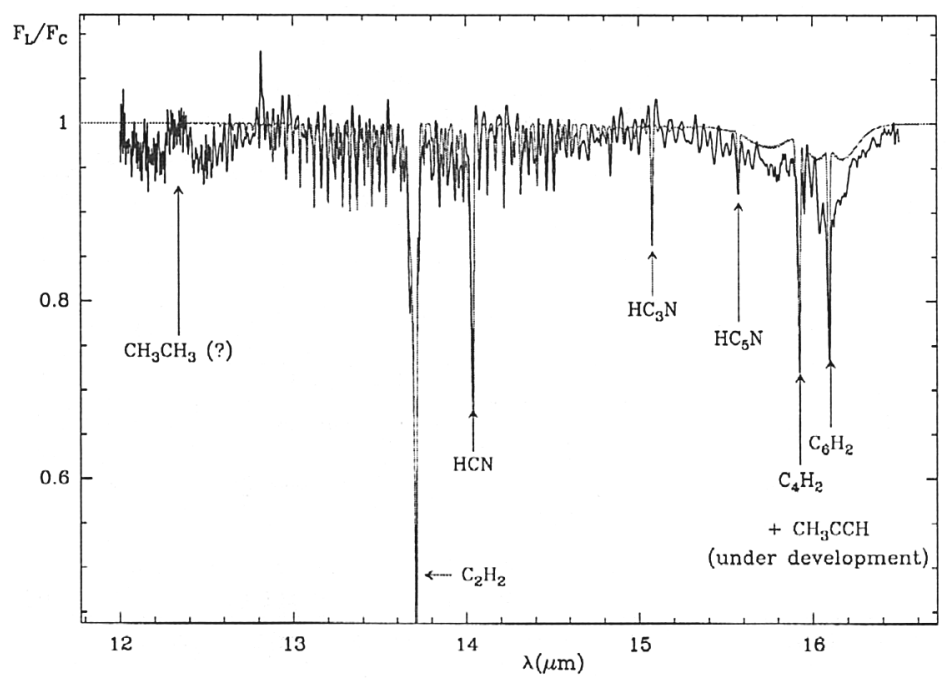

Figure 6. The SWS spectrum of CRL618 around $15 \mu \mathrm{m}$. The narrow features around $16 \mu \mathrm{m}$ are due to $\mathrm{C}_{4} \mathrm{H}_{2}$ and $\mathrm{C}_{6} \mathrm{H}_{2}$.

Figure 6 shows the spectrum of CRL618 around $15 \mu \mathrm{m}$ which has to be compared with that of IRC+10216 shown in Figure 1. The spectra are completely different. Only the fundamental bending modes of $\mathrm{C}_{2} \mathrm{H}_{2}$ and $\mathrm{HCN}$ are detected. In addition, the spectrum presents several narrow features corresponding to the Q-branches of the bending modes of $\mathrm{HC}_{3} \mathrm{~N}, \mathrm{HC}_{5} \mathrm{~N}$ and, for the first time in interstellar space, to the bending modes of $\mathrm{C}_{4} \mathrm{H}_{2}$ and $\mathrm{C}_{6} \mathrm{H}_{2}$. These two molecular species have strong combination bands around $8 \mu \mathrm{m}$ that are very prominent in the SWS spectrometer data - see Cernicharo et al. (1999b). The lowest energy bending modes of $\mathrm{HC}_{3} \mathrm{~N}$ and $\mathrm{C}_{4} \mathrm{H}_{2}$ are also detected at $45 \mu \mathrm{m}$ with the LWS spectrometer.

The column densities of these species are around (5-10) $10^{16} \mathrm{~cm}^{-2}$, i.e., only a factor of $10-20$ below that of $\mathrm{C}_{2} \mathrm{H}_{2}$. The corresponding abundance ratio in IRC +10216 between $\mathrm{C}_{2} \mathrm{H}_{2}$ and $\mathrm{C}_{4} \mathrm{H}_{2}$ is larger than 100 , which shows that important physical processes have modified the chemistry of CRL618. Moreover, and in agreement with the millimeter wave observations, the $\mathrm{HC}_{3} \mathrm{~N}$ and $\mathrm{HC}_{5} \mathrm{~N}$ $\mathrm{Q}$-branches are not detected in IRC+10216, which indicates the very large abundances of these species in CRL618. $\mathrm{C}_{4} \mathrm{H}_{2}$ and $\mathrm{C}_{6} \mathrm{H}_{2}$ have also been detected in CRL2688, another proto-planetary nebula.

In addition to these species, $\mathrm{NH}_{3}$ produces an impressive forest of absorption bands around $10 \mu \mathrm{m}$. These bands are blended with those from $\mathrm{C}_{2} \mathrm{H}_{4} \cdot \mathrm{CH}_{4}$, $\mathrm{C}_{3} \mathrm{H}_{2}$ and a combination band of $\mathrm{C}_{2} \mathrm{H}_{2}$ also contributes to the absorption bands around $8 \mu \mathrm{m}$ (Cernicharo et al. 1999b). The ISO-SWS spectrum of CRL618 is dominated by a very large number of absorption bands that remain unidentified. Many of them could arise from small hydrocarbons like $\left(\mathrm{CH}_{3}\right)_{2},\left(\mathrm{CH}_{3}\right)_{2} \mathrm{CH}_{2}$, $\mathrm{CH}_{3}, \mathrm{CH}_{2}, \mathrm{CH}_{3} \mathrm{CCH}$, etc. We are working on the identification of these features, but an important input from laboratory data is needed. 


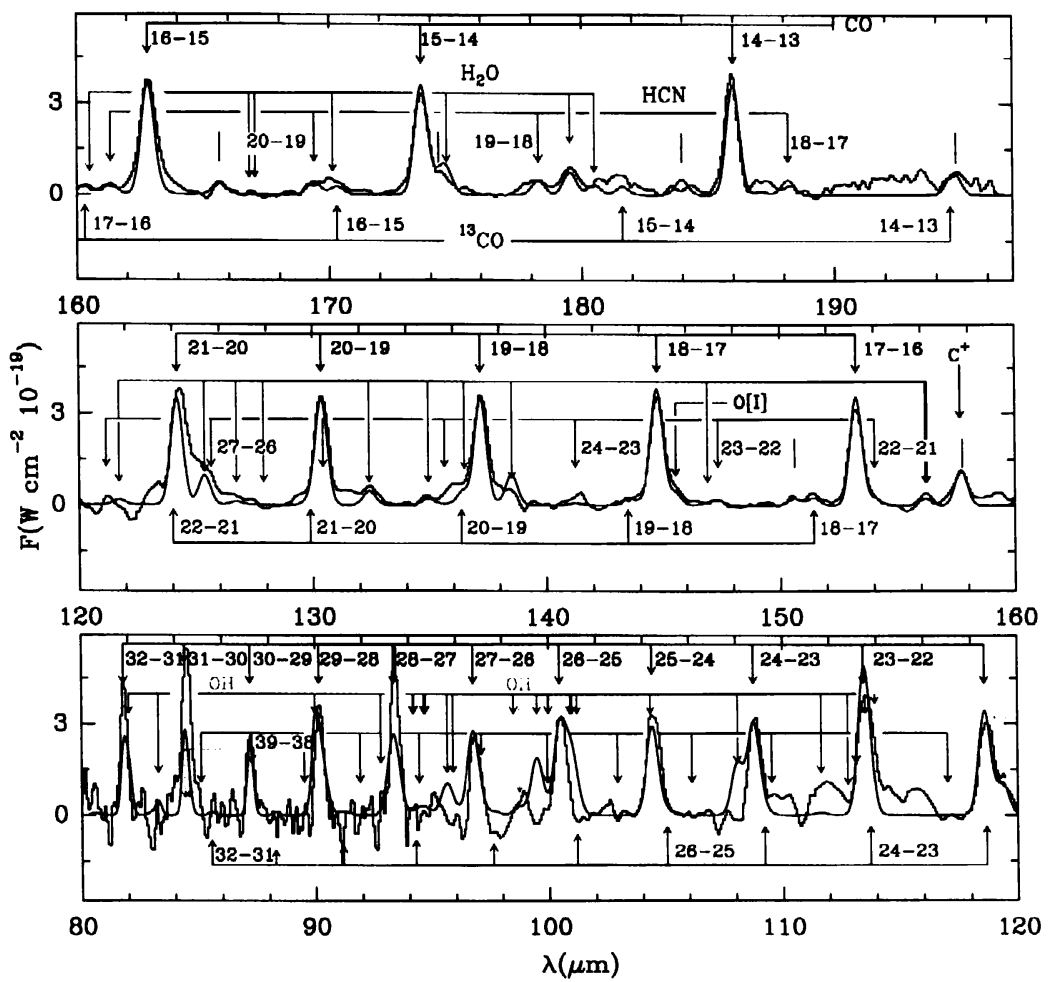

Figure 7. Continuum subtracted ISO-LWS spectra of CRL618. The result of a multispatial component model is shown by the continuous line. The lines of $\mathrm{CO},{ }^{13} \mathrm{CO}, \mathrm{HCN}, \mathrm{H}_{2} \mathrm{O}$ and $\mathrm{OH}$ are indicated by arrows while those of HNC are indicated by vertical lines (from $J=22-21$ at $150.627 \mu \mathrm{m}$ to $J=17-16$ at $194.759 \mu \mathrm{m}$ ) (from: Herpin \& Cernicharo 1999).

Herpin \& Cernicharo (1999) have analysed the LWS spectrum of CRL618. In addition to the lines of $\mathrm{CO},{ }^{13} \mathrm{CO}, \mathrm{HCN}$ and $\mathrm{HNC}$, they report the detection of $\mathrm{H}_{2} \mathrm{O}$ and $\mathrm{OH}$ emission together with the fine structure lines of [OI] at 63 and $145 \mu \mathrm{m}$ (see Figure 7). The derived abundances for these O-bearing species, relative to $\mathrm{CO}$, are $410^{-2}, 810^{-4}$ and 4.5 .

From an analysis of the structure of the envelope, and using an LVG model, they have obtained a reasonable fit to the data (see Figure 7). They suggest that O-bearing species other than $\mathrm{CO}$ are produced in the innermost region of the circumstellar envelope. The UV photons from the central star, together with the high velocity winds prevailing in CRL618, destroy most of the molecular species produced in the AGB phase and allow a chemistry dominated by standard ionneutral reactions. Not only do these reactions allow the formation of O-bearing species but they also modify the abundances of C-rich molecules like HCN and HNC for which we found an abundance ratio of $\simeq 1$, much lower than in AGB stars. Hence, CRL618 shows an enormous variety of carbon chains and small hydrocarbons together with O-bearing species like $\mathrm{H}_{2} \mathrm{O}, \mathrm{OH}, \mathrm{HCO}^{+}$and $\mathrm{H}_{2} \mathrm{CO}$. 

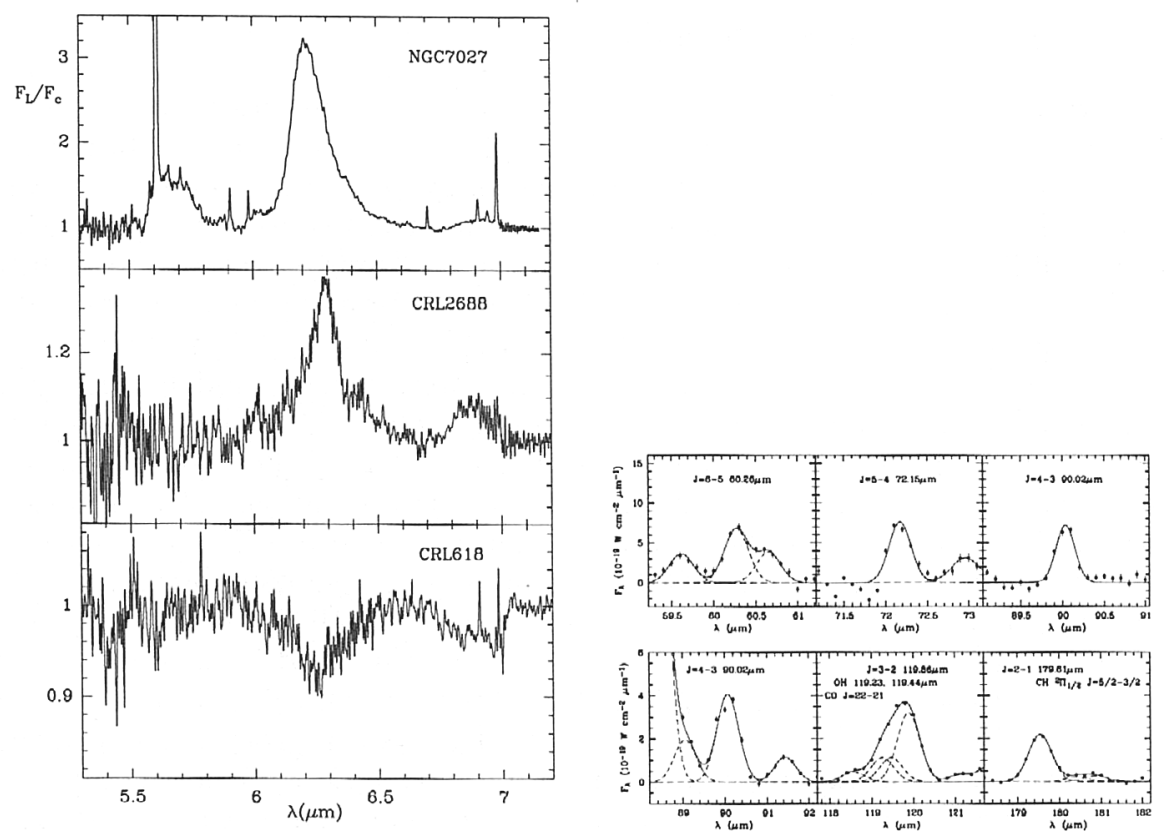

Figure 8. (right panels) The SWS spectrum of NGC7027, CRL2688 and CRL618 around $6 \mu \mathrm{m}$. A baseline, resulting from a fit to the continuum emission, has been removed from the three spectra. (left panels) $\mathrm{CH}^{+}$lines observed in NGC7027. The solid lines are Gaussian line profile fits to the observed features (for blends, contributions from individual components are shown as dashed lines). The $J=2 \rightarrow 1$ line at $179.62 \mu \mathrm{m}$ was previously assigned to $\mathrm{H}_{2} \mathrm{O}$ (from: Cernicharo et al. 1997).

Finally, the comparison of the spectrum of NGC7027, CRL2688 and CRL618 at $6 \mu \mathrm{m}$ (see Figure 8a) indicates that the region where the UIBs appear in emission is, in the case of CRL618, dominated by several absorption bands with clear vibrational structure. Although we have not found yet potential carriers for the broad absorption around $6 \mu \mathrm{m}$, we believe that it is produced by several carbonrich gas phase molecules of moderate size. The detection of long carbon chains and small hydrocarbons in CRL618 and CRL2688 suggests that in the protoplanetary stage of C-rich stars, the extremely high velocity winds associated with the evolution of these objects, and the strong UV field from the central star, could modify the chemistry of the CSE. A large variety of small molecules are formed during this stage. They could be the pieces from which large carbonrich macromolecules could form and produce the UIBs through excitation by UV photons. Alternatively, all these species, including the carriers of the UIBs, could be released to the gas phase from the dust grains under the action of the strong shocks, and of the UV photons, to which the innermost envelope is submitted. 


\section{1. $\mathrm{CH}^{+}$in Planetary Nebulae}

$\mathrm{CH}^{+}$was one of the first molecules detected in astrophysics through its ${ }^{1} \Pi-$ ${ }^{1} \Sigma$ electronic transition at optical wavelengths. This molecule is abundant in the diffuse interstellar medium and during many years the chemical reactions involved in its formation have been subject of important controversy. Cernicharo et al. (1997) have reported the discovery of several pure rotational transitions of $\mathrm{CH}^{+}$in the LWS spectrum of NGC7027 (see Figure 8b). The strong line at $179.62 \mu \mathrm{m}$ (nearly coincident in frequency with the $2_{12}-1_{01}$ transition of water vapour) and the lines at 119.90 and $90.03 \mu \mathrm{m}$ (reported as unidentified by Liu et al. 1996), whose frequencies are in harmonic relation $2: 3: 4$, arise from the $J=2 \rightarrow 1,3 \rightarrow 2$, and $4 \rightarrow 3$ rotational transitions of $\mathrm{CH}^{+}$. This identification is strengthened by the detection in the LWS spectra of the next two rotational lines of $\mathrm{CH}^{+}$, at 72.17 and $60.22 \mu \mathrm{m}$. Cernicharo et al. (1997) have derived a rotational temperature for the $\mathrm{CH}^{+}$lines of $160 \mathrm{~K}$, a volume density of a few $10^{7} \mathrm{~cm}^{-3}$, and a $\mathrm{CH}^{+} / \mathrm{CO}$ abundance ratio of $(0.2-1) 10^{-3}$. Given the carbonrich nature of NGC7027 and the strong UV radiation field in its inner regions it is thus not too surprising to find $\mathrm{CH}^{+}$in this prototypical PN. However, this is the first time that $\mathrm{CH}^{+}$has been seen through its pure rotational spectrum. Taking into account the dipole moment of $\mathrm{CH}^{+}(1.7 \mathrm{D})$ and the energy of its rotational levels, the rotational transitions of this molecular species constitute a unique tool to trace the physical conditions of the PDRs of the interstellar and circumstellar medium.

\section{Conclusions}

ISO has opened a new view of the near-, mid- and far-IR spectra of evolved stars providing useful constraints to the modelling of the CSEs of these objects. An important conclusion from the modelling of these data is the crucial role of radiative pumping in populating the vibrational levels of molecular species. However, three important caveats still have to be addressed: (i) the absolute lack of collisional cross sections for the vibrational levels of most molecular species; (ii) the lack of laboratory data for the spectroscopy of most molecules of astrophysical interest, and (iii) ISO's poor spectral resolution which cannot separate the different spatial contributions to the emission/absorption features produced by the molecules. The latter one will be solved when the FIRST satellite will fly in 2007. The HIFI heterodyne instrument that will be installed onboard FIRST will have a very high spectral resolution $\left(\Delta \lambda / \lambda\right.$ better than $\left.10^{-6}\right)$, excellent frequency coverage $(0.5-3 \mathrm{THz})$ and very high sensitivity allowing the observation of less abundant species and the derivation of accurate line profiles for $\mathrm{CO}, \mathrm{HCN}, \mathrm{H}_{2} \mathrm{O}$ and other molecular species. To solve the two first problems, however, we have to establish close collaborations with scientists doing quantum-mechanical calculations and laboratory spectroscopy work. Molecular Astrophysics is a multidisciplinary science and ISO is showing the importance of close collaboration among different physics and chemistry groups to derive the physical conditions of the gas in CSEs and in any warm environment of the interstellar medium. 
Acknowledgments. I thank Spanish DGES and CICYT for support under grants PB96-0883 and ESP98-1351.

\section{References}

Aoki, W., Tsuji, T., \& Ohnaka, K. 1999, A\&A, 350, 945

Barlow, M.J., et al. 1996, A\&A, 315, L241

Cernicharo, J. 1998, Astr. Space Science, 255, 303

Cernicharo, J., Barlow, M., González-Alfonso, E., et al. 1996, A\&A, 315, 201

Cernicharo, J., Gottlieb, C.A., Guélin, M., et al. 1989a, ApJ, 341, L25

Cernicharo, J., Gottlieb, C.A., Guélin, M., et al. 1991a, ApJ, 368, L39

Cernicharo, J., Gottlieb, C.A., Guélin, M., et al. 1991b, ApJ, 368, L43

Cernicharo, J. \& Guélin, M. 1987, A\&A, 183, L10 1996, A\&A, 309, L27

Cernicharo, J., Guélin, M., \& Kahane, C., 2000, A\&A, in press

Cernicharo, J., Guélin, M., Menten, K., \& Walmsley, C.M. 1987b, A\&A, 181, L1

Cernicharo, J., Guélin, M., Peñalver, J., et al. 1989b, A\&A, 222, L20

Cernicharo, J., Guélin, M., \& Walmsley, C.M. 1987a, A\&A, 172, L5

Cernicharo, J., Kahane, C., Gómez-González, J., \& Guélin, M. 1986a, A\&A, $164, \mathrm{~L} 1$

1986b, A\&A, 167, L5

Cernicharo, J., Liu, X.-W., González-Alfonso, E., et al. 1997, ApJ, 483, L65

Cernicharo, J., Yamamura, I., González-Alfonso, E., et al. 1999a, ApJ, 526, L21

Cernicharo J., et al. 1999b, in The Universe as seen by ISO, eds. P. Cox \& M.F. Kessler (ESA SP-427, March 1999, Paris), 285

Clegg, P.E., et al. 1996, A\&A, 315, L38

Cox, P., González-Alfonso, E., Barlow, M., et al. 1996, A\&A, 315, L265

de Graauw Th., et al. 1996, A\&A, 315, L49

González-Alfonso, E. \& Cernicharo, J. 1997, A\&A, 322, 938

1999a, in The Universe as seen by ISO, eds. P. Cox \& M.F. Kessler

(ESA SP-427, March 1999, Paris), 325

1999b, ApJ, 525, 845

González-Alfonso, E., Cernicharo, J., van Dishoeck, E.F., et al. 1998, ApJ, 502, L169

Guélin, M., Cernicharo, J., Kahane, C., \& Gómez-González, J. 1986, A\&A, 157, L17

Guélin, M., Cernicharo, J., Kahane, C., Gómez-González, J., \& Walmsley, C.M. 1987, A\&A, 175, L5

Guélin, M., Cernicharo, J. \& Travers, M.J. 1997, A\&A, 317, L1

Guélin, M., Lucas, R. \& Cernicharo, J. 1993, A\&A, 280, L19

Guélin, M., Neininger, N. \& Cernicharo, J. 1998, A\&A, 335, L1

Herpin, F. \& Cernicharo, J. 1999, ApJ Letters, submitted.

Ishii, K., Hirano, T., Nagashima, U., Weis, B., \& Yamashita, K. 1993, ApJ, 410, L43

Justtanont, K., de Jong, T., Helmich, F.P., et al. 1996, A\&A, 315, L217

Justtanont, K., de Jong, T., Helmich, F.P., et al. 1998, A\&A, 330, L17

Keady, J.J. \& Hinkle, K.H. 1988, ApJ, 331, 539

Keady, J.J. \& Ridway, S.T. 1993, ApJ, 406, 199

Lucas, R. \& Cernicharo, J. 1989, A\&A, 218, L20 
Liu, X.-W., Barlow, M.J., Nguyen-Q-Rieu, et al. 1996, A\&A, 315, L257 Matsuura, M., Yamamura, I., Murakami, H., et al. 1999, A\&A, 348, 579 Neri, R., et al. 1992, A\&A, 262, 544

Neufeld, D.A., Chen, W., Melnick, G.J., et al. 1996, A\&A, 323, L237

Ryde, N., Schoier, F.L., \& Olofsson, H. 1999a, A\&A, 345, 841

Ryde, N., Eriksson, K., \& Gustafsson, B. 1999b, A\&A, 341, 579

Suzuki, H., Ohishi, M., Kaifu, N., Ishikawa, S.I., \& Kasuga, T. 1986, PASJ, 38, 911

Sylvester, R.J., Barlow, M.J., Liu, X.-W., et al. 1997, MNRAS, 291, L42

Truong-Bach, Sylvester, R.J., Barlow, M.J., et al. 1999, A\&A, 345, 925

Tsuji, T. 1973, A\&A, 23, 411

Tsuji, T., Ohnaka, K., Aoki, W., \& Yamamura, I. 1997, A\&A, 320, L1

Turner, B., Steimle, T.C., \& Meerts, L. 1994, ApJ, 426, L97

Wiedemann, G.R., Hinkle, K.H., Keady, J.J., et al. 1991, ApJ, 382, 321

Woitke, P., Helling, Ch., Winters, J.M., \& Jeong, K.S. 1999, A\&A, 348, L17

Yamamura, I., de Jong, T., \& Cami, J. 1999a, A\&A, 348, L55

Yamamura, I., de Jong, T., Onaka, T., Cami, J., et al. 1999b, A\&A, 341, L9

Ziurys, L., Apponi, A.J., Cernicharo, J., \& Guélin, M. 1995, ApJ, 445, L47

\section{Discussion}

A. Lapinov: (1) What fraction of C-stars has HCN $J=2-1$ masers? (2) What is the efficiency of IR pumping: what is the ratio of photons at the maser HCN $J=2-1$ transition to IR pumping photons? (3) Did you try to detect the maser effect in HNC?

J. Cernicharo: (1) We observed $\sim 40$ objects. Masers are observed in $25 \%$. (2) I am not ready to answer this question now. (3) Not yet.

$U$.G. Jørgensen: Comment: very simplified models of the stellar atmosphere can often fit the spectra very well, but at the cost that we loose understanding of the star. The "3-layer-models" you refer to for analysing ISO spectra have at least 7 free parameters. They hide the very complicated structure of the photosphere that ISO spectra analysed with our fully self-consistent models reveal (e.g. Jørgensen, Hron, \& Loidl 2000, A\&A, in press, about the $14 \mu \mathrm{m}$ band in carbon stars). 